\title{
LA RUTA DEL VINO DEL SOMONTANO DE BARBASTRO (HUESCA) COMO PROYECTO TERRITORIAL DE IDENTIDAD CULTURAL
}

\author{
Ana Castelló Puig ${ }^{1}$ y M. ${ }^{a}$ Luz Hernández Navarro \\ 1 Dpto. de Geografía y Ordenación del Territorio, Universidad de Zaragoza \\ Facultad de Ciencias Humanas y de la Educación \\ castello@unizar.es \\ 2 Dpto. de Geografía y Ordenación del Territorio, Universidad de Zaragoza \\ Facultad de Filosofía y Letras \\ mlhernan@unizar.es
}

\begin{abstract}
Resumen: En este trabajo se presenta el producto "Ruta del Vino Somontano" de la comarca aragonesa de la que toma su nombre en Barbastro (Huesca), como un Proyecto Territorial con identidad cultural, que sirve de referente dentro y fuera del marco relacional en el que se ubica. El elemento aglutinante de todas las dimensiones de la identidad es el vino y el enoturismo la actividad principal, aprovechando los recursos patrimoniales del territorio y gracias a una buena acción de gobernanza como elemento principal.
\end{abstract}

Palabras clave: Proyecto Territorial, Recursos Patrimoniales, enoturismo, gobernanza.

\begin{abstract}
In this paper, the product "Route of the Somontano Wine", from the area of Barbastro (Huesca) in the region of Aragón, is presented. This is a territorial project with cultural identity which serves as a benchmark within and outside the relational context in which is located. Wine is the glue holding all the dimensions of identity, and wine tourism is the main activity; heritage resources from the territory are considered, thanks to a good governance action as the main element.
\end{abstract}

Keywords: Project Planning, Heritage Resources, wine tourism, governance.

Recibido: 6-5-11. Aceptado: 2-2-12. 


\section{Introducción}

La actual manifestación del capitalismo en sus diversas expresiones, generalizadas con el nombre de economía global en el contexto de la revolución tecnológica y con el conocimiento como base fundamental, presenta variados escenarios, en los que las relaciones entre las empresas, las instituciones, las personas y el entorno han adquirido nuevas dimensiones. Han surgido otras formas relacionales, es posible también establecer la complementariedad entre lo económico y lo extraeconómico y operar con nuevas pautas de comportamiento en una escala local perfectamente compatible con la global. Además, las actuaciones, desde la perspectiva del paradigma territorial acorde con el modelo de desarrollo endógeno, favorecen la consideración de una nueva ruralidad, en cuyo análisis se vuelven muy útiles algunos instrumentos conceptuales elaborados por la economía industrial y regional para el estudio de los Sistemas Productivos Locales (Fonte, 2006).

Desde los años noventa del pasado siglo las actuaciones que se ponen en marcha en el ámbito local de nuestro entorno en materia de desarrollo, evidencian la necesidad de actuar con estrategias de diferenciación, que necesitan de la acción colectiva, entre lo público y lo privado. Los territorios se presentan como agentes muy activos en la consecución de las identidades territoriales y proyectan una imagen de reputación y reconocimiento, que favorece la competitividad y añade valor a todos los productos que se ofertan desde el mismo.

En este entorno innovador compiten los territorios y el éxito depende de su capacidad para saber aprovechar y revalorizar los recursos reales y potenciales materiales e inmateriales. La adaptación a las nuevas reglas del juego se pone mejor en práctica en los lugares de proximidad; es decir en las localidades, que desempeñan un papel muy activo como agentes de desarrollo. Se supera así la función anterior de meros contenedores de actividades y servicios y se asume la actual, que las convierte en protagonistas del desarrollo y portadoras de responsabilidades añadidas, para conseguir la competitividad territorial global que se espera de ellas. Los productos territoriales han de ser competitivos y generar ingresos, para así cumplir la función social de mejorar el nivel de vida de los habitantes locales, sin perder los criterios de universalidad, que han de traspasar las fronteras de forma que se logre explotar la identidad territorial en los mercados globales (Aranda y Combariza, 2007).

La Identidad Territorial se configura a través de un proceso de interacción continua entre lo social, lo temporal y lo espacial, en una construcción colectiva, a partir de elementos específicos del territorio, que participan en el diseño de una imagen y una marca de producto diferenciada, que añade valor a todos los demás.

En este contexto, el objetivo de este trabajo es presentar el caso de la "Ruta del Vino Somontano" en la comarca aragonesa de la que toma su nombre, como un 
Proyecto Territorial, derivado de la conjunción de acciones individuales y colectivas, públicas y privadas, que han hecho compatibles las estrategias propias de un sector industrial, dinámico y competitivo, basado en la industria vitivinícola, con la participación en acciones de desarrollo que han dado lugar a la generación de un Proyecto Territorial con identidad cultural, que ha servido de referente dentro y fuera del marco relacional en el que se ubica. El elemento aglutinante de todas las dimensiones de la identidad es el vino, como producto específico e identificador de todos los demás. La industria vitivinícola participa a través de la Denominación de Origen Somontano que, junto con el Ayuntamiento de Barbastro y la Comarca del Somontano, han elaborado el Proyecto Territorial, aprovechando todos los recursos patrimoniales del territorio, considerado agente activo, espacio construido, interiorizado e identificado por toda la sociedad y gestionado desde la proximidad local.

Esto ha sido posible por la existencia de una serie de factores que, aparentemente inconexos, han operado cada vez más interrelacionados y de manera simultánea. Todos han sido importantes, pero ha sido la calidad de la gobernanza la que ha hecho posible que se consiguiera la competitividad territorial. Para presentarlo, exponemos en primer lugar la vinculación de la vid y el vino en la comarca, a continuación, el camino recorrido por sus habitantes en el contexto del nuevo paradigma de desarrollo endógeno, en el siguiente apartado, las acciones de gobernanza territorial que se han adoptado para que cristalizara con éxito y, finalmente, el proyecto "Ruta del Vino Somontano" con los agentes implicados.

\section{La vinculación del vino y la viña con el Somontano de Barbastro}

Los vestigios históricos del cultivo de la vid y la industria vitivinícola en el Somontano de Barbastro dan cuenta de su existencia en época de los griegos, fenicios y romanos, pero será en la Edad Media cuando el paisaje promiscuo de vides y olivos se pueda generalizar y constatar como una de las características definitorias del Somontano de Barbastro (Rodrigo, 2001), en plena armonía con el almendro. Es la típica trilogía mediterránea, perfectamente adaptada a la orografía del terreno propio del pie de sierra, con pendientes pronunciadas y ambiente climatológico propicio debido a la altitud a la que están los cultivos. Conforme avanzan los siglos, la calidad atrae empresarios y comerciantes foráneos que se instalan en la ciudad, Barbastro, contribuyendo así a configurar un tejido industrial y comercial que genera una cultura y una mentalidad de las gentes construida y cultivada sobre el intercambio desde antiguo, por ser paso obligado entre la montaña y el llano, el este y el oeste; por ello en un contacto permanente con el exterior, que traspasa los límites administrativos de la misma. La evolución de la producción y de la extensión superficial del viñedo reflejan los cambios propiciados por la influencia de la crisis de la filoxera en Francia 
y en España, que afectó a las empresas pioneras de principios del siglo pasado, seguido de la falta de apoyo institucional para su cultivo y los vaivenes propios de un producto que ha pasado, de carecer de interés, a ejercer un monopolio entre los cultivos y ser considerado en función más de su calidad que de la cantidad. A las tres bodegas iniciales de principios de siglo, se suma en los años sesenta la creación de la Cooperativa Comarcal de Somontano de Sobrarbe y se inicia así una apuesta decidida por la calidad y el inicio de los trámites para conseguir la Denominación de Origen, que se hace efectiva en el año 1984. La creación del Consejo Regulador y las buenas prácticas son un elemento de atracción empresarial para autóctonos y foráneos y el vino se ha convertido en un fetiche identificador de la cultura autóctona de la zona. Su vinculación es total con el entorno y el prestigio en su elaboración a lo largo de la cadena de producción lo tiene ganado en el contexto de la Denominación de Origen.

Actualmente son 34 las bodegas que forman parte de la Denominación de Origen Somontano, de las cuales seis, las de mayor producción (de 1.000 .000 a 6.000 .000 botellas/año), son las que lideran el proyecto "Ruta del Vino Somontano" y en todas ellas el arte y la cultura presiden el diseño de su arquitectura, sus etiquetas, su entorno y sus acciones complementarias, bajo el lema "el arte del vino y el enoturismo". En el tamaño medio hay catorce bodegas cuyo distintivo es "la suma de lo propio"; son empresas familiares (entre 50.000 y 500.000 botellas/año), cada una con su toque personal, su propia historia y filosofía. Las catorce restantes, de dimensiones más pequeñas, con una fabricación inferior a las 50.000 botellas/año son de reciente creación, pero con tradición de varias generaciones en el cultivo de la vid. Se caracterizan por su funcionalidad y por tener unas señas de identidad muy arraigadas en el territorio, con vinculaciones estrechas con lo local, que se materializan en el nombre de la marca y, en general, en el diseño que utilizan en toda su iconografía.

La identificación de las empresas con la marca Somontano se puede deducir a través de muchos indicadores, pero uno de ellos, muy significativo, es la relación que tienen sus marcas con el entorno. El 61\% de las marcas de vino que salen al mercado están relacionadas con nombres que tiene que ver con lo local, lo endógeno, bien sea nombres geográficos del medio natural, identificaciones con el nombre de los parajes donde se cultivan las viñas, expresiones únicas de usos, costumbres, tradiciones, en definitiva territorio.

Las señas de identidad son las propias de la cultura mediterránea y es muy oportuno que el nombre geográfico "Somontano" haya sido el instrumento idóneo para generar confianza e identificación por parte de la población local y extralocal y para transmitir información relacionada con la calidad, el buen hacer y la reputación de todos los productos de la comarca. 
El conocimiento, la identificación, las prácticas, las rutinas, etc., todo lo que tiene que ver con la vid y el vino, está perfectamente interiorizado en los habitantes y vinculado al conocimiento tácito, que es el que favorece y permite la comunicación dentro del sistema (Fonte, 2006). La cultura del vino se ha convertido en recurso susceptible de proporcionar valor añadido a la economía local, como un producto económico exportable sólo o en combinación con otros formando paquetes turísticos en las diferentes épocas del año. Se generan así economías externas para las empresas del vino y para los territorios.

Todos los actores locales que participan en el proyecto (empresas, agentes sociales, instituciones etc.) obtienen beneficios materiales e inmateriales, derivados de la revalorización integral del nombre geográfico de todo el territorio asociado al vino. La reputación de la marca Somontano va ya intrínsecamente unida a la calidad en cualquier producto, inspira confianza en el consumidor y, por lo tanto, reduce de manera drástica el nivel de asimetría informativa que habitualmente preside la relación producción-consumo (Arfini, 2006). Sin la protección y los recursos de organización y de reputación que proporciona la marca colectiva sería muy difícil para la mayoría de las empresas y servicios artesanales de la zona poder competir en el mercado.

En este proceso todos participan y todos obtienen beneficios y para garantizar que no existe discriminación en ningún sentido y evitar las interferencias de agentes internos o externos que pudieran malograr los objetivos se ha elegido el vino como elemento identitario del Proyecto. De esta forma, en el interior, la población y los territorios implicados en el citado Proyecto Territorial, se sienten identificados sin fisuras y, al mismo tiempo, se gana la consideración en el exterior, porque la marca "Somontano" es suficientemente conocida.

\section{El escenario del desarrollo. Las lecciones aprendidas en la comarca}

En la Comarca del Somontano la filosofía del modelo endógeno es conocida sobradamente porque han participado, desde la década de los noventa, en los proyectos de la Unión Europea implementados con el método LEADER. Saben lo que supone elaborar un proyecto de territorio que contemple la competitividad territorial, porque han recorrido el tránsito desde Leader II a Leader Plus, han trabajado en la Agenda 2000, en varias Iniciativas Comunitarias y en otras estrategias de desarrollo. El salto cualitativo ha sido fundamental para adquirir conciencia clara de lo que para un territorio supone ser competitivo y capaz de soportar la competencia del mercado, garantizando al mismo tiempo la viabilidad medioambiental, económica, social y cultural y aplicando lógicas de red y de articulación interterritorial. Han asumido claramente la idea de la multifuncionalidad de la empresa agraria, de la cualificación 
y diferenciación de los productos agroalimentarios y del territorio mismo, en una estrategia que tiene más en cuenta la calidad que la cantidad.

De ahí que resulte más fácil avanzar en la construcción del Proyecto Territorial, porque la parte más inmaterial del mismo, la que afecta a las relaciones sociales, tiene ya un largo recorrido. Se ha instalado en la mentalidad colectiva la visión del desarrollo en todas sus dimensiones, superando la tradicional lógica funcional y sectorial del crecimiento. Han interiorizado el papel productivo, residencial, ambiental y relacional que propone la Unión Europea, principalmente a partir de la Estrategia Territorial Europea, diversificando las actividades para mantener un paisaje agrario vivo, activo competitivo y con atractivo cultural. La simbiosis entre el patrimonio cultural, el natural y el económico, actuando de manera conjunta y activa, contribuye a la venta del territorio como un todo integrado (ETE, 1999).

Han experimentado un proceso endógeno, global e integrado que se materializa o sustenta en redes de intercambio que generan un diálogo entre todos los actores o agentes basado en la confianza, la cooperación y la competencia, con una meta y objetivos claros, fruto del ejercicio de autonomía de cada una de las partes, lo que les otorga credibilidad en la participación. En el desarrollo local, los territorios toman la iniciativa y todos están invitados a participar (Vachon, 2001).

Como demuestran Gumuchian et al (2003), Joyal (2002) y Arocena (2001), citados por Klein (2005), esta nueva manera de hacer desarrollo corresponde a sistemas de actores sociales, políticos y económicos; es decir, a sistemas de acción. Las empresas y el resto de actores que actúan, aúnan la cercanía locacional, o proximidad espacial y la proximidad relacional. En la base de este desarrollo endógeno se identifican: la sinergia entre los distintos protagonistas del territorio, una dinámica positiva de dimensiones económica, social y medioambiental, cierta visión de perspectivas a largo plazo y presencia de capital social para consolidar los esquemas de colaboración (Noya, 2007). En esta perspectiva diversos autores demuestran la existencia de sistemas territoriales en los que priman mecanismos locales de regulación y formas territoriales de innovación (Aydalot, 1986).

\section{La gobernanza como elemento principal}

Las estrategias locales de revalorización requieren la elaboración de un marco de referencia organizativo y de gestión, que configura una forma de gobernanza capaz de dirigir y organizar el proceso y, a través de él, el uso y la distribución de los beneficios resultantes de la acción de revalorización por parte de los distintos actores locales (Arfini, 2006). 
En el Somontano de Barbastro, la acción de gobernanza es ejercida por distintos colectivos locales que configuran una red, creada a iniciativa del Ayuntamiento y la Comarca que, a la proximidad espacial, han unido una manera de actuar, de relacionarse y de concebir juntos la revalorización del territorio. El objetivo específico es construir un producto, la "Ruta del Vino Somontano", desde su propia identidad, facilitar la comercialización conjunta de todos los componentes implicados y garantizar la competitividad y calidad del producto al consumidor.

Estas acciones han supuesto una garantía en el proceso de distribución de los beneficios entre todos los territorios de la comarca, porque no se trata de una gobernanza sectorial o corporativa que prime los intereses de una empresa o de un sector solamente; se trata de una gobernanza territorial que permite y facilita la participación de la representación democrática de todos los colectivos públicos y privados, que han centrado su actuación en la protección y revalorización del territorio desde una dimensión integral, evitando acciones de privilegio y de oportunismo interesado desde alguna perspectiva individual, de personas o empresas. El acierto en la gobernanza permite que, entre las muchas ventajas conseguidas, exista la garantía de la distribución de los beneficios debido a la utilización por parte de todos los partícipes del proyecto, de la marca territorial ligada al vino. No obstante, como señalan algunos autores, se puede demostrar la existencia de otros elementos (Sylvander y Bajolle, 2000; Paccciani y cols. 2001, citados por Arfini (2006), que inciden igualmente en los resultados y que están presentes en el Somontano: la tipología del producto típico, su vinculación con la comunidad local, la estructura del sector y la existencia de empresas foráneas dominantes, la existencia de un marco institucional adecuado y los recursos económicos, paisajísticos, culturales y medioambientales de la zona considerada.

La acción de gobernanza se consolida con el respeto, la cooperación y la colaboración entre las Instituciones públicas y la iniciativa privada, lo que no es un obstáculo para ejercer acciones de control, con el fin de mantener altos niveles de calidad para conseguir la reputación colectiva. Este es el elemento clave de una gobernanza fuerte, capaz de adoptar políticas decididas que conllevan la realización de acciones encaminadas a preservar y revalorizar la reputación del producto final; en este caso el proyecto "Ruta del vino Somontano".

\section{El Proyecto Territorial "Ruta del vino Somontano"}

Una "Ruta del Vino" es, principalmente, un producto y un destino turístico de gran calidad, adherido al proyecto "Rutas del Vino de España", liderado por la Asociación Española de Ciudades del Vino (ACEVIN), bajo la tutela del Ministerio de Industria, Turismo y Comercio, a través de la Secretaría General de Turismo y del Ministerio de 
Medio Ambiente y Medio Rural y Marino (MARM) y con vínculos relacionales con la Red Europea de Ciudades del Vino (RECEVIN). La misión principal es valorizar el potencial endógeno de las zonas donde el cultivo de la viña y la elaboración del vino han sido la base productiva tradicional de su desarrollo; tienen que estar ubicadas en zonas protegidas por denominación de calidad de sus vinos y contar con una población mínima de 5.000 habitantes. El principal objetivo es potenciar el sector como motor de otras actividades (turismo, promoción económica, empleo, protección del medio natural, etc.). La participación en la Ruta exige unos estándares de calidad y unas normas de autorregulación muy estrictas, contempladas en el Manual del Producto Turístico Rutas del Vino de España, donde se refleja el sistema de certificación y los registros de gestión, señalización, promoción y comercialización que deben cumplir todos los establecimientos (ACEVIN, 2010).

Bajo el concepto temático de la vitivinicultura, las rutas del vino, integran de forma modular, los recursos y servicios turísticos de interés de las DOs, con el fin de construir un producto desde su propia identidad. En el Somontano de Barbastro se inician los trabajos en el año 2004, con la elaboración de la "Ruta del Vino Somontano", una de las trece que existen en España, cuyo ente gestor y responsable es la Asociación de Promoción Turística del Somontano, entidad sin ánimo de lucro, creada en el año 2005 por el Ayuntamiento de Barbastro, la Comarca del Somontano y el Consejo Regulador de la Denominación de Origen Somontano. El proyecto definitivo se aprueba en 2006 y pasa a formar parte de las "Rutas del Vino de España" y, por lo tanto, de la oferta internacional a través de los canales establecidos por la Secretaría General de Turismo. Se caracteriza por la originalidad, la profesionalización de los servicios orientada a la innovación, la diversificación y, sobre todo, la no masificación y el marchamo de gran calidad, certificada y avalada con periodicidad anual por agentes externos. Es un proyecto integral, técnicamente muy complejo, en torno al vino y su cultura. El eje temático es el vino y el enoturismo sintetiza los valores del patrimonio, el paisaje, la gastronomía, la cultura y las costumbres y tradiciones populares, a través de un conjunto de servicios ofertados de manera conjunta. La complejidad se deriva, principalmente, de la necesidad de aunar esfuerzos y establecer redes de cooperación interempresariales e interinstitucionales, que en este caso ha sido más fácil de conseguir por las acciones de gobernanza que se han puesto en práctica, tal como hemos expresado en el apartado anterior. Se caracteriza por la variedad de sus componentes, la calidad homogénea de los mismos, la adaptabilidad a las necesidades del consumidor y la diferenciación de sus propuestas. Es un Proyecto Territorial construido desde la propia identidad, lo que facilita la comercialización conjunta del territorio y garantiza la competitividad y calidad del producto final (Asociación de Promoción Turística del Somontano, 2011). El predominio del vino se deja notar en la pertenencia al proyecto de un número mayoritario de establecimientos relacionados con el mismo (bodegas), incluso algunos de ellos procedentes de las comarcas colindantes, que se han adherido utilizando sus lazos de pertenencia a la Denominación de Origen Somontano. El vino 
y su cultura están presentes a lo largo de toda la cadena de valor del producto global ofertado en la ruta. En torno al mismo se aglutinan los elementos imprescindibles para considerarlo como Proyecto Territorial: una selección cuidada de bodegas, establecimientos y servicios turísticos (alojamientos, restaurantes, bares, comercios especializados, agencias de viajes, empresas de transporte) y una rica oferta de ocio (museos, deportes de aventura, spas urbanos, centros de interpretación), de tal modo que la implicación, colaboración y cooperación entre las empresas es significativa.

En el proyecto participan 85 establecimientos que tienen certificación con sello de calidad, elemento indispensable y requisito imprescindible exigido en las reglas establecidas por los agentes, a través de una fuerte gobernanza ejercida por los mismos. La diversidad de actividades ofertadas es pareja a la respectiva de empresas participantes ya que se han adherido a la Ruta un alto porcentaje de empresas de la zona: diecisiete bodegas (el $50 \%$ del total), cuatro empresas de deportes de aventura (18\% del total comarcal), siete centros de actividad turística, museos y centros de interpretación ( $46 \%$ de los existentes ), tres empresas de transporte y cuatro agencias de receptivo (100\% del total), una de ellas denominada Enodestino.com, especializada en enoturismo y encargada de gestionar especialmente los paquetes turísticos de la ruta, diecinueve restaurantes ( $25 \%$ del total), tres bares exclusivos de vinos (100\% del total) once hoteles y hostales (39\% del total) nueve casas de turismo rural (20\% del total), además de varios comercios especializados en productos típicos de la comarca y dos empresas agroalimentarias.

La implicación territorial es amplia porque afecta a 26 núcleos de población de la comarca, que representan el $45 \%$ del total y a dos de las comarcas colindantes en las que se extiende la Denominación de Origen Somontano; asimismo, genera dos puestos de trabajo directo, fijos completos y permanentes y varios indirectos a través de las actividades que promueve. Desde los inicios, el nivel de participación se ha incrementado, ya que han pasado de 34 establecimientos adheridos en 2006, a 64 en 2008 y a 85 en 2010 y actualmente hay un listado de establecimientos pendientes de aceptación, previa verificación de los altos niveles de calidad que la pertenencia a la Ruta exige.

La oferta, diferente para cada estación del año, contiene diversas actividades, pero siempre con el vino como telón de fondo: catas de vino y de aceite, talleres de vino y gastronomía, visitas guiadas a las bodegas y centros de interpretación, juegos enogastronómicos, cursos de fotografía entre viñedos y bodegas, vinoterapia, rutas de senderismo por las sierras y parajes del entorno natural y cultural, etc.

Es un claro ejemplo de diversificación de la economía rural, que oferta los productos y el territorio Somontano de Barbastro como objeto de consumo, transformado y revalorizado en paisaje cultural con gran valor patrimonial, lo que ha contribuido a desestacionalizar el turismo, porque es objeto de atención e interés los 
trescientos sesenta y cinco días del año (Asociación de Promoción Turística Somontano, 2009).

En este modelo territorial es importante destacar el comportamiento activo y la dinámica empresarial del sector vitivinícola. De una parte, las bodegas, han introducido innovaciones en la producción, puestas de manifiesto en diversos aspectos, desde la tecnología utilizada en las diferentes fases de la misma, hasta el consumo final, pasando por las pautas de localización y, sobre todo, por las nuevas relaciones de proximidad, entre el mundo económico y la sociedad civil, que se manifiestan en diversas actuaciones conjuntas, en las interrelaciones con las demás empresa y con las redes internacionales (Fernández, 2000). Y, por otra parte, su participación, junto con las instituciones locales como ya hemos apuntado anteriormente, en la creación del Proyecto Territorial Ruta del Vino Somontano, desde los inicios en el año 2005. No se trata solamente de una tarea filantrópica o de mero patrocinio, la inmersión de las empresas en las nuevas formas de desarrollo es de igual a igual, colaborando, constituyendo el capital social y contribuyendo a la gobernanza para conseguir más y mejor desarrollo territorial. La dimensión integral de la responsabilidad social empresarial se manifiesta en una empresa no sólo por lo que hace, sino también por lo que es para un conjunto de personas que se relacionan con ella (Moreno, 2007). En nuestro caso, las empresas vitivinícolas son un sello de identidad de la comarca y suponen el orgullo de los habitantes que las tienen interiorizadas en su subconsciente como algo propio, lo que contribuye a engrandecer su territorio, convertido en recurso y elemento activo y da lugar a la generación de ventajas, no solo comparativas sino también competitivas (Caravaca, et al., 2003) donde se implementan acciones de desarrollo local.

A las acciones culturales y de mecenazgo, desarrolladas tradicionalmente, se ha unido la participación en el proyecto piloto "Hacia un modelo de competitividad, sostenibilidad y gobernanza en el Medio Rural' liderado por el MARM, de tal forma que la "Ruta del Vino Somontano" se ha convertido en "Territorio Enoturístico Socialmente Responsable" concebido, según el presidente de la Asociación de Promoción Turística del Somontano, como "una berramienta de trabajo y compromiso para que Somontano se consolide como un territorio que conjuga el equilibrio entre los aspectos económicos, sociales, ambientales y culturales del modo de vida local que implica a los colectivos, entidades y asociaciones" (Huguet, 2010).

En este contexto innovador el desarrollo se analiza muy bien desde el enfoque que proporciona la metodología de trabajo adaptada a la corriente teórica de las convenciones, que pone el énfasis en las relaciones y en las formas en que éstas se constituyen: la economía como relaciones, el proceso como conversación y coordinación, los agentes del proceso como actores humanos y reflexivos y la naturaleza de la acumulación económica como activos relacionales (Storper, 1998). 
Las nuevas maneras de actuar han dado lugar a la generación de características distintivas, más relacionadas con la calidad que con la cantidad de los productos elaborados. Se ha introducido una nueva lógica en el sistema de relaciones a lo largo de todo el proceso de producción, que siguiendo a Storper (1998) consiste en la búsqueda de cómo afrontar la incertidumbre a través de la coordinación entre diferentes agentes, como requisito indispensable para promover eficiencia y competitividad en el sistema económico. La rapidez en la gestación del Proyecto Territorial y los resultados aceptables que se están constatando, se debe, en parte, al aprendizaje que se ha producido en el territorio en la manera de competir, cooperar, compartir, etc., que permite adquirir variados compromisos y moverse con comodidad y superar el aislamiento que en ocasiones lleva consigo la ruralidad.

Tal y como han demostrado Aparicio, Sánchez, Alonso y Rodero (2008), las Denominaciones de Origen se constituyen en un marco institucional propicio para la construcción de un entorno innovador y bajo su paraguas la innovación social y la económica se conjugan en un territorio bien delimitado. La regulación no innova por sí sola, pero crea las condiciones adecuadas como consecuencia de la interacción reiterada entre los agentes locales.

\section{Conclusiones}

La "Ruta del Vino Somontano" favorece la adaptación de la oferta de los productos de la comarca a las exigencias de la demanda, gracias a los atributos de calidad y a la reputación reconocida al vino de la marca Somontano, que aglutina en torno a su nombre gran variedad de elementos con identidad territorial que por sí solos no podrían conseguir la competitividad en el mercado.

Existe una estrecha convergencia entre el sistema productivo vitivinícola, el territorio, las instituciones públicas, los agentes privados y los recursos endógenos y exógenos, para establecer sinergias con la finalidad de conseguir un desarrollo económico, social y medioambiental.

El redescubrimiento de este recurso ha contribuido a favorecer la autoestima, el sentido de pertenencia, el fomento de la territorialidad y el establecimiento de redes de acción en el territorio. Y el vino se ha convertido en un elemento de cohesión social. El nombre geográfico del Somontano aglutina en sí mismo el pasado, el presente y el futuro; extiende los beneficios, la reputación y el marchamo de calidad a todos los sectores y acciones, beneficiando así a todo el territorio. En la construcción de esta seña de identidad ha sido imprescindible contar con la descentralización administrativa y la capacidad de decisión que ha asumido la comarca, a través del proceso de comarcalización, y que se ha convertido en una de las dimensiones principales del desarrollo. 


\section{Bibliografía}

Acevin (2010). Ciudades del vino, en www.ciudadesdelvino.com/ (Consulta: 16.01.2011)

Aparicio, J. Sánchez, J.L., Alonso, J.L. y Rodero, V. (2008). La Ribera del Duero, geografía de un medio innovador en torno a la vitivinicultura, Scripta Nova, 277, http://www.ub.es/geocrit/sn/sn-277.htm (Consulta: 19.02.2009).

Aranda, Y., Combariza, J., (2007). Las marcas territoriales como alternativa para la diferenciación de productos rurales en www.rimisp.org/FCKeditor/userfiles/docs /pdf/DTR-IC/las-marcasterritoriales.pdfl (Consulta: 16.11.2010).

Arfini, F. (2006): Productos típicos y desarrollo rural: entre calidad y políticas de gobernanza, Estudios Agrosociales y Pesqueros, 13-38.

Asociación de Promoción Turística del Somontano (2011). Ruta del vino. Somontano vive el vino, en http:/www.rutadelvinosomontano.com (Consulta: 19.01.2011).

Aydalot, Ph. (1986). Milieux innovateurs en Europe. Paris, GREMI

Caravaca, I. González, G. y Silva, R (2003). Redes e innovación socio-institucional en sistemas productivos locales, Boletín de la Asociación de Geógrafos Españoles, 36, 103-115.

Comisión Europea (1999). Estrategia Territorial Europea, Luxemburgo.

Fernández, Mo Jo (2000).Reestructuración productiva y desarrollo regional, Estudios Regionales, 58, 45-77.
Fonte, M. (Coord.) (2006). Desarrollo rural e identidad cultural: reflexiones teóricas y casos empíricos, en http://www.rimisp. org/getdoc.php?docid=5102 (Consulta: 16.11.2010)

Huguet, A. (2010). La Ruta del Vino ya es territorio socialmente responsable, Diario del Altoaragón, 7 de noviembre, p. 16.

Klein, J.L. (2005). Iniciativa local y desarrollo: respuesta social a la globalización neoliberal, Eure, 94, 25-39 www.scielo.php?pid $=\mathrm{S} 0250 \ldots$ script . (Consulta: 4.03.2010).

Moreno, CH. (2007). Definición del concepto de responsabilidad social empresarial. En Hernández, M. A., Losada, A. y Macías, A. (Coords.). Estrategia y conducta social de la organización, Salamanca, Universidad Pontificia de Salamanca, pp. 17-28.

Noya, A. (2007). Desarrollo local integrado y economía social. Cuadernos de Economía Social, Madrid, CEPES.

Rodrigo, M $\mathrm{a}^{\mathrm{a}}$ L. (2001). Viñedo y vino en el Somontano de Barbastro: los siglos medievales, en Sabio, A. (Coord.). Vino de Siglos en el Somontano de Barbastro. Una bistoria social y cultural: las vidas desde las viñas, Barbastro (Huesca), Consejo Regulador de la Denominación de Origen Somontano, pp. 15-44.

Storper, M. (1998). Las economías regionales como activos relacionales. Ekonomiaz, 1045 .

Vachon, B. (2001). El desarrollo local. Teoría y práctica, Gijón, Ediciones Trea, S.L. 